\title{
Risk Stratification of Bethesda III Category Thyroid Nodules
}

\author{
Eiman Alseddeeqi ${ }^{\mathrm{a}, \mathrm{f}}$, Rawia Mubarak ${ }^{\mathrm{b}}$, Ajda Altinoz ${ }^{\mathrm{c}}$, \\ Yogesh More ${ }^{\mathrm{d}}$, Abdulghani Elomami ${ }^{\mathrm{e}}$
}

\begin{abstract}
Background: Atypia of undetermined significance/follicular lesion of undetermined significance (AUS/FLUS) carries a malignancy risk reaching up to $50 \%$. Based on the reported malignancy rate in a given population, the clinical practice towards such a category varies. We hereby identify clinical parameters for risk stratification to aid in decision making for either surgical referral or a clinical follow-up. Our aim is to calculate the malignancy risk in Bethesda category III and to identify clinical parameters that guided both clinicians and patients at our institutions to reach a clinical decision.
\end{abstract}

Methods: A retrospective review of patients with Bethesda category III thyroid nodules from the tertiary centers in the Emirate of Abu Dhabi during January 2011 through December 2015 was carried out. Malignancy risk in Bethesda category III nodules and repeat fineneedle aspiration (FNA) utility were calculated. Parameters guided referral to surgery were studied.

Results: A total of 202 cases were included in the study. Of these, 101 cases underwent surgery initially following the first FNA, and 10 cases following repeat FNA. Histology confirmed malignancy in $41 \%$ of cases that went initially to surgery, and in $40 \%$ of cases that underwent a repeat FNA. Repeat FNA resulted in 17 (44.74\%) cases being re-classified into benign category, 10 (26.3\%) being AUS/FLUS category, six (15.7\%) being both suspicious and malignant, and five $(13.16 \%)$ being unsatisfactory. Repeating FNA resulted in a definitive diagnostic utility in $50 \%$ of the cases.

Conclusions: The relatively high malignancy rate in our institutions, the suspicious radiographic features and the repeat FNA predictive value stratified Bethesda category III nodules for appropriate referrals to surgery.

Manuscript submitted November 16, 2019, accepted November 25, 2019

${ }^{a}$ Division of Endocrinology, Sheikh Khalifa Medical City, Abu Dhabi, UAE bDivision of Clinical Services, Sheikh Khalifa Medical City, Abu Dhabi, UAE 'Division of General Surgery, Tawam Hospital, Al Ain, Abu Dhabi, UAE ${ }^{\mathrm{d}}$ Division of Otorhinolaryngology, Sheikh Khalifa Medical City, Abu Dhabi, UAE

eDivision of Anatomy Pathology and Cytopathology, Tawam Hospital, Al Ain Abu Dhabi, UAE

${ }^{f}$ Corresponding Author: Eiman Alseddeeqi, Division of Endocrinology, Sheikh Khalifa Medical City, PO Box 51900, Abu Dhabi, UAE.

Email: ealseddeeqi@seha.ae

doi: https://doi.org/10.14740/jem625
Keywords: AUS/FLUS; Thyroid FNA; Thyroid cancer; Bethesda category III

\section{Introduction}

Bethesda category III thyroid nodules which includes atypia of undetermined significance/follicular lesion of undetermined significance (AUS/FLUS), has a 5-15\% risk for thyroid malignancy $[1,2]$. The Bethesda system was introduced to establish diagnostic categories for thyroid aspirates and to imply malignancy risks for clinical management recommendations [3]. Therefore, the recommended guideline for the initial management of AUS/ FLUS is a clinical follow-up by repeating fine-needle aspiration (FNA) in $\geq 3$ months based on the risk of thyroid malignancy in a given population [4]. Repeating FNA resulted in re-classification of $70 \%$ of Bethesda III samples into Bethesda II category [5]. The utility of repeating FNA for category III was therefore an auxiliary tool for clinical decision making. Bethesda category III nodules remained a similar category in up to $31 \%$ of cases [6]. Repeating an FNA may result in a cost-effective practice by avoiding unnecessary diagnostic surgery. If other studies are appraised, one would find varying reported rates of malignancy risks ranging from $2 \%$ to $50 \%$, which will dictate the clinical practice; i.e. repeating an FNA versus referring to surgery [4, 7-12].

Two meta-analyses concluded higher malignancy rates than that reported by the Bethesda [13, 14]. Possible reasons for this discrepancy include differences in patients' demographics, technical issues which include the adequacy of the sampled material, the optimization of cellular preservation and slide preparation, and the level of comfort/expertise of the reporting pathologist. Molecular markers testing has been recommended by the American Thyroid Association to provide a pretest probability for Bethesda III nodules. The results of such testing provide guidance regarding whether to repeat a biopsy or to refer to surgery [15].

The rate of malignancy for Bethesda category III in a given population is an essential fact to delineate the next step of management. There is no uniform practice in the Emirate of Abu Dhabi towards such a category as there is little known regarding thyroid cancer behavior in the UAE population.

The American Thyroid Association recognizes that the prevalence of malignancy for the indeterminate categories varies substantially among centers, and states that "it is crucial to know the prevalence of malignancy within each indeterminate cytological category at one's institution". Based on that state- 
ment, our study results can provide a recommendation regarding managing thyroid nodules with Bethesda category III and assist in counselling patients to reach a decision at our institutions [15]. A similar commentary in Thyroid states the same principle, "It is crucial to know the incidence of malignancy in each of the Bethesda categories in one's own institution in order to make reasonable treatment decisions" [16]. The McGill thyroid nodule score has served as a predictor tool for malignancy in thyroid nodules [17]. A study published in 2015 has studied that clinical tool to evaluate the incidence of malignancy in indeterminate nodules preoperatively [18].

Therefore, we chose to address factors that risk-stratify the Bethesda category III nodules in order to aid in clinical decision, i.e. referral to surgery vs. repeat an FNA at our centers. Our objectives are to calculate the malignancy rate, examine the medical practice towards such a category and predict the clinical utility of repeating an FNA.

\section{Materials and Methods}

\section{Study settings and participants}

This is a retrospective medical records review of patients with Bethesda category III nodules from three tertiary centers in the Emirate of Abu Dhabi. All Bethesda category III cases that were diagnosed from January 2011 through December 2015 were extracted from the electronic pathology database. The database identified 240 patients above 14 years of age, of which 38 patients other than Bethesda category III were excluded. The FNA biopsy was carried out under ultrasound guidance. The diagnosis of Bethesda category III was made by different certified pathologists at the three different institutions. The total number of patients ended up being included in the study was 202. An institutional research board approval was obtained from each institution.

\section{Study variables}

Information on patients' gender, age at time of FNA procedure was extracted from the electronic medical records. Age was categorized into three groups: younger than 40 years old, 40 - 64 years old and 65 and above years old. Clinical management of the Bethesda category III cases was recorded, and it included one of the followings: diagnostic surgery or follow-up with either a repeat FNA (in $3+$ months from the first one) or an ultrasound (in $6+$ months). Surgery was either a thyroid lobectomy or a total thyroidectomy. Forty-nine cases had no follow-up or surgery as an initial management. Patients with no follow-up included patients who visited other medical institutions in the same city to have a second opinion, those who travelled elsewhere for a second opinion, and a portion whom we did not have any date about. Fourteen patients chose to follow up with ultrasound despite recommendations for either surgery or an FNA repeat. The ultrasound was performed 1 year from the last FNA and demonstrated no increase in nodule size (a significant growth was defined as a growth of $>20 \%$ increase in two nodule dimensions or $>50 \%$ increase in the volume). The clinical follow-up included follow-up with an ultrasound. A major indication for surgery was the presence of suspicious features on ultrasound. Patients' preferences were another indicator explaining such a high rate for surgical referrals. Radiation exposure and a positive family history for thyroid malignancy were other indicators for referring to surgery at our institutions.

A certified general pathologist diagnosed cases of Bethesda category III based on adequate number of thyroid follicles on FNA smears. Hospital onsite adequacy assessment was performed by a cytology screener, who performed slides preparation. Conventional air dried and alcohol fixed smears were prepared and subject to Diff-Quik and Papanicolaou stains, respectively. Diagnosing Bethesda category III was based on the 2007 National Cancer Institute Thyroid fine needle aspiration state of the science conference criteria $[1,15]$.

Following up by either an ultrasound or a repeat FNA was proposed if there were no suspicious ultrasonographic features such as calcifications and a nodule appearing taller-than-wide. On some occasions, patients' preferences were guiding the clinical decision. The repeat FNA was performed 3 months or more from the initial FNA.

\section{Statistical analysis}

The malignancy rate was calculated for nodules that underwent thyroid lobectomy or total thyroidectomy. Those without follow-up were not included in the analyses of malignancy risk calculation. Descriptive statistics were represented in tables.

Data were tabulated, analysed, and figures were made using Microsoft Excel version 15.33.

\section{Results}

A total of 202 cases out of all thyroid FNAs performed during 2011 to 2015 were diagnosed as AUS/FLUS (Fig. 1). Of these, $166(82.2 \%)$ were women and $36(17.8 \%)$ were men. The age of the patients ranged from 14 to 84 years with median age of 47 years (Table 1). Fourteen patients had follow-ups with ultrasound. Forty-nine patients did not have any follow-up. One hundred and eleven out of 202 patients with AUS/FLUS category underwent surgery; 101 immediately after the first FNA and 10 following the repeated FNA (Fig. 2). One hundred and one patients $(50 \%)$ underwent immediate surgery. Their histology confirmed 42 malignant (41\%) cases and 59 benign (59\%) cases. Thirty-eight out of the 202 patients (18.8\%) underwent a repeat FNA procedure. The repeat procedure resulted in 17 (44.74\%) cases being re-categorised as benign, $10(26.3 \%)$ as AUS/FLUS, six (15.7\%) as both suspicious and malignant, and five (13.16\%) as unsatisfactory (Fig. 3). Ten cases from the repeat FNA group underwent surgery. Three out of four suspicious cases and one out of two malignant cases were confirmed to be malignant following surgery (40\%). The repeat FNA resulted in definitive diagnostic utility for $50 \%$ of the cases; and $44.74 \%$ of the nodules were re-classified into Bethesda category II upon FNA repeat. 


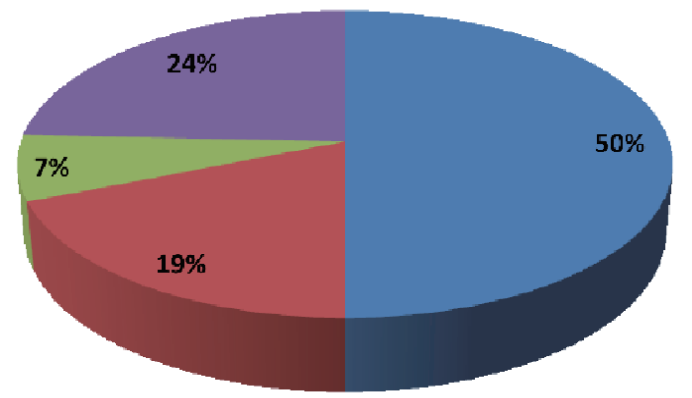

- Immediate surgery

repeated FNA

Follow up with Thyroid US, no significant changes

Without follow up

Figure 1. Total number of patients with AUS diagnosis and management. FNA: fine-needle aspiration; AUS: atypia of undetermined significance.

Table 1. Baseline Demographics of Patients

\begin{tabular}{llll} 
& Tawam Hospital & Shiekh Khlifa Medical City and Mafraq Hospital & Total \\
\hline Male & 18 & 18 & 36 \\
Female & 98 & 68 & 54 \\
$<40$ years & 34 & 20 & 166 \\
$40-64$ years & 73 & 56 & 129 \\
$\geq 65$ years & 49 & 10 & 19 \\
\hline
\end{tabular}

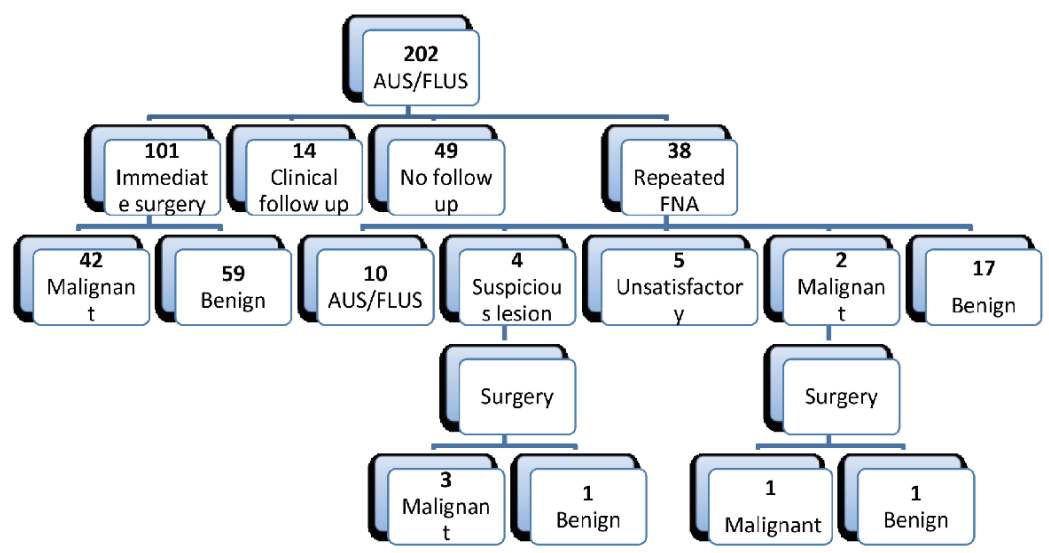

Figure 2. Number of all patients with AUS and management outcomes. AUS/FLUS: atypia of undetermined significance/follicular lesion of undetermined significance; FNA: fine-needle aspiration.

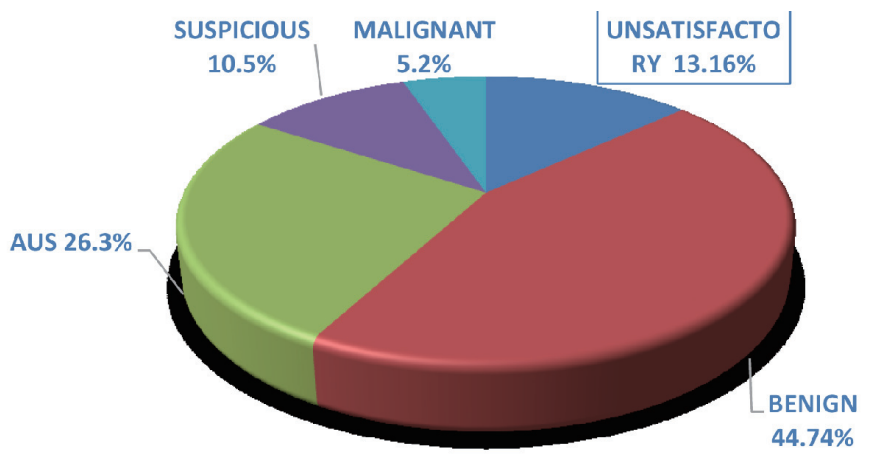

Figure 3. Diagnosis of repeated FNA cases. FNA: fine-needle aspiration; AUS: atypia of undetermined significance. 


\section{Discussion}

The reported malignancy risk in Bethesda category III varies among different institutions. Factors including inter and intraobserver variability of reporting among pathologists affect that variation [19].

In our study, the overall number of cases with Bethesda category III was 202 cases amongst all biopsies performed during the 5 -year period. Our malignancy rate is $41 \%$ for cases that underwent surgery initially and $40 \%$ for cases that underwent surgery following a repeat FNA. This is a significantly higher rate than that reported by the Bethesda. A similar study performed in our city published in 2018 , concluded a lower rate which was $20 \%$ [20]. Since only $50 \%$ of patients underwent surgery, it is possible that the true malignancy rate in AUS/ FLUS nodules is just slightly above $20 \%$. Still that higher risk is explained by the fact that our three institutions are tertiary centers, and probably higher risk patients are being referred to there. Globally the incidence of thyroid cancer is rising [21]. In $\mathrm{UAE}$, thyroid cancer is the second most common malignancy amongst females [22]. On the same note, a study conducted by Alseddeeqi et al concluded a high malignancy rate among all thyroid nodules in the UAE [23]. Patient's genetics make up and the nature of the life style in our region might have played a role in such a finding.

Ozluk et al reported a lower malignancy rate $(36 \%)$ compared to ours in their study of 3,444 nodules [24]. Ho et al reported malignancy rate that is also lower compared to ours $(37.8 \%)$ in their population who presented to a tertiary cancer center where a referral bias could have been existed [16]. A retrospective analysis by Lee et al, involving 4,933 aspirates was consistent with our malignancy rate $(41.2 \%)$ in their Bethesda category III cases [25]. The malignancy rate in our institutions is a fact clinicians can base their decisions on along with the following parameters: the clinical presentation, ultrasonographic characteristics and nuclear features [26].

The recommendation for AUS/FLUS result category is a repeat FNA in 3 months. Fifty percent of our AUS/FLUS cases underwent surgery following the first FNA. A major indication for surgery was the presence of suspicious features on ultrasound. Patients' preferences were another indicator explaining such a high rate for surgical referrals. Compared to another study, the percentage of cases underwent surgery initially was $61 \%$, a rate which is higher than ours. That study's major cause for surgical referral was the presence of suspicious features on ultrasound [20]. Therefore, the rate of malignancy in a given institution, the clinical presentation, and nuclear features need to be considered along with the ultrasound findings when calculating the pretest probability in AUS/FLUS cases [14]. Radiation exposure and a positive family history for thyroid malignancy were other indicators for referring to surgery at our institution. The relatively affordable cost for surgery by the insuring agent in our institutions was in favor of recommending surgery over implementing a costly molecular markers assessment.

Repeat FNA provided a definitive diagnosis in only $50 \%$ of the cases according to our study. This result is of value for patients as they are involved in the decision making. Our result provided a better utility than a study published by Allen et al, where the repeat FNA procedure provided a $40 \%$ definitive diagnosis [27]. Yet our utility rate is not considered to be of guidance to clinicians and patients for the decision making. Our malignancy rate in cases underwent surgery initially is similar to that in cases underwent surgery following a repeat FNA. That finding is similar to the one found by Vanderlaan et al, where their AUS/FLUS cases demonstrated similar malignancy rate following initial and repeat FNA [28].

Based on our malignancy rate, the availability and the cost of surgery, the presence of suspicious features on ultrasound or a positive clinical indicator, surgery was favored as an option in our cohort. The utility of repeating an FNA was another factor that favored referrals to surgery.

The limitations in our study are that of any retrospective study design. We had 49 cases without follow-ups. More than one certified pathologist was involved in diagnosing AUS/ FLUS category which could affect the rate of Bethesda category III diagnosis, but that was somehow overcame by adhering to AUS/FLUS diagnostic criteria which kept equivocal results to a minimum. Since malignancy rates reported were only for nodules that underwent resection, following up the non-surgical nodules would rule out false negative results. Moreover, the false negative results could have been verified if surgery was performed on nodules with Bethesda category II. If those 14 cases with radiological follow-up were further studied at a longer time period (over 1 year), a true malignancy rate would have been estimated.

This is the first study of its kind in the region that addressed multiple factors to risk-stratify Bethesda category III nodules. Our results definitely would guide clinicians and patients referred to our institutions to the next plan of management. The diagnostic utility for repeat FNA was a useful tool for patients to guide them for decision making.

We believe that our study makes a significant contribution to the care provided in our tertiary centers because it takes into consideration readily available clinical data for risk stratification. Based on the findings in our tertiary centers, we were able to properly refer those nodules to surgery.

\section{Conclusions}

The relatively high malignancy rate in our institutions, the presence of suspicious radiographic features and the repeat FNA predictive value guided both clinicians and patients for appropriate referrals to surgery.

\section{Acknowledgments}

None to declare.

\section{Financial Disclosure}

The study did not receive any form of financial support. 


\section{Conflict of Interest}

None to declare.

\section{Informed Consent}

Not applicable.

\section{Author Contributions}

EA, RM, AE contributed to the study design. RM and AE accomplished data collection. AE analysed the data. EA, AA, and YM drafted different parts of the manuscript and all approved the final version.

\section{Abbreviations}

UAE: United Arab Emirates; AUS/FLUS: atypia of undetermined significance/follicular lesion of undetermined significance; FNA: fine-needle aspiration

\section{References}

1. Cibas ES, Ali SZ. The Bethesda System For Reporting Thyroid Cytopathology. Am J Clin Pathol. 2009;132(5):658665.

2. Kayilioglu SI, Dinc T, Sozen I, Senol K, Katar K, Karabeyoglu M, Tez M, et al. Thyroid nodules with atypia or follicular lesions of undetermined significance (AUS/ FLUS): analysis of variables associated with outcome. Asian Pac J Cancer Prev. 2014;15(23):10307-10311.

3. Liu X, Medici M, Kwong N, Angell TE, Marqusee E, Kim MI, Larsen PR, et al. Bethesda Categorization of Thyroid Nodule Cytology and Prediction of Thyroid Cancer Type and Prognosis. Thyroid. 2016;26(2):256-261.

4. Brandler TC, Aziz MS, Coutsouvelis C, Rosen L, Rafael OC, Souza F, Jelloul FZ, et al. Young investigator challenge: Atypia of undetermined significance in thyroid FNA: Standardized terminology without standardized management - a closer look at repeat FNA and quality measures. Cancer Cytopathol. 2016;124(1):37-43.

5. Erivwo P, Ghosh C. Atypia of Undetermined Significance in Thyroid Fine-Needle Aspirations: Follow-Up and Outcome Experience in Newfoundland, Canada. Acta Cytol. 2018;62(2):85-92.

6. Na DG, Kim JH, Sung JY, Baek JH, Jung KC, Lee H, Yoo $\mathrm{H}$. Core-needle biopsy is more useful than repeat fineneedle aspiration in thyroid nodules read as nondiagnostic or atypia of undetermined significance by the Bethesda system for reporting thyroid cytopathology. Thyroid. 2012;22(5):468-475.

7. Bongiovanni M, Krane JF, Cibas ES, Faquin WC. The atypical thyroid fine-needle aspiration: past, present, and future. Cancer Cytopathol. 2012;120(2):73-86.
8. Kholova I, Ludvikova M. Thyroid atypia of undetermined significance or follicular lesion of undetermined significance: an indispensable Bethesda 2010 diagnostic category or waste garbage? Acta Cytol. 2014;58(4):319329.

9. Gan TR, Nga ME, Lum JH, Wong WM, Tan WB, Parameswaran R, Ngiam KY. Thyroid cytology-nuclear versus architectural atypia within the "Atypia of undetermined significance/follicular lesion of undetermined significance" Bethesda category have significantly different rates of malignancy. Cancer Cytopathol. 2017;125(4):245-256.

10. Park HJ, Moon JH, Yom CK, Kim KH, Choi JY, Choi SI, Ahn SH, et al. Thyroid "atypia of undetermined significance" with nuclear atypia has high rates of malignancy and BRAF mutation. Cancer Cytopathol. 2014;122(7):512-520.

11. Sullivan PS, Hirschowitz SL, Fung PC, Apple SK. The impact of atypia/follicular lesion of undetermined significance and repeat fine-needle aspiration: 5 years before and after implementation of the Bethesda System. Cancer Cytopathol. 2014;122(12):866-872.

12. Kim TH, Jeong DJ, Hahn SY, Shin JH, Oh YL, Ki CS, Kim JW, et al. Triage of patients with AUS/FLUS on thyroid cytopathology: effectiveness of the multimodal diagnostic techniques. Cancer Med. 2016;5(5):769-777.

13. Krauss EA, Mahon M, Fede JM, Zhang L. Application of the Bethesda Classification for Thyroid Fine-Needle Aspiration: Institutional Experience and Meta-analysis. Arch Pathol Lab Med. 2016;140(10):1121-1131.

14. Valderrabano P, Khazai L, Thompson ZJ, Leon ME, Otto KJ, Hallanger-Johnson JE, Wadsworth JT, et al. Cancer risk stratification of indeterminate thyroid nodules: a cytological approach. Thyroid. 2017;27(10):1277-1284 .

15. Haugen BR, Alexander EK, Bible KC, Doherty GM, Mandel SJ, Nikiforov YE, Pacini F, et al. 2015 American Thyroid Association Management Guidelines for adult patients with thyroid nodules and differentiated thyroid cancer: the American Thyroid Association Guidelines task force on thyroid nodules and differentiated thyroid cancer. Thyroid. 2016;26(1):1-133.

16. Ho AS, Sarti EE, Jain KS, Wang H, Nixon IJ, Shaha AR, Shah JP, et al. Malignancy rate in thyroid nodules classified as Bethesda category III (AUS/FLUS). Thyroid. 2014;24(5):832-839.

17. Sands NB, Karls S, Amir A, Tamilia M, Gologan O, Rochon L, Black MJ, et al. McGill Thyroid Nodule Score (MTNS): "rating the risk," a novel predictive scheme for cancer risk determination. J Otolaryngol Head Neck Surg. 2011;40(Suppl 1):S1-13.

18. Varshney R, Forest VI, Mascarella MA, Zawawi F, Rochon L, Hier MP, Mlynarek A, et al. McGill Thyroid Nodule Score - does it help with intermediate thyroid nodules? J Otolaryngol Head Neck Surg. 2015;44(1):2.

19. Iskandar ME, Bonomo G, Avadhani V, Persky M, Lucido D, Wang B, Marti JL. Evidence for overestimation of the prevalence of malignancy in indeterminate thyroid nodules classified as Bethesda category III. Surgery. 2015;157(3):510-517.

20. Khalil AB, Dina R, Meeran K, Bakir AM, Naqvi S, Al 
Tikritti A, Lessan N, et al. Indeterminate Thyroid Nodules: A Pragmatic Approach. Eur Thyroid J. 2018;7(1):39-43.

21. Bray F, Ferlay J, Soerjomataram I, Siegel RL, Torre LA, Jemal A. Global cancer statistics 2018: GLOBOCAN estimates of incidence and mortality worldwide for 36 cancers in 185 countries. CA Cancer J Clin. 2018;68(6):394424.

22. La Vecchia C, Malvezzi M, Bosetti C, Garavello W, Bertuccio P, Levi F, Negri E. Thyroid cancer mortality and incidence: a global overview. Int $\mathrm{J}$ Cancer. 2015;136(9):2187-2195.

23. Alseddeeqi E, Baharoon R, Mohamed R, Ghaith J, AlHelali A, Ahmed LA. Thyroid malignancy among patients with thyroid nodules in the United Arab Emirates: a five-year retrospective tertiary Centre analysis. Thyroid Res. 2018;11:17.

24. Ozluk Y, Pehlivan E, Gulluoglu MG, Poyanli A, Salmaslioglu A, Colak N, Kapran Y, et al. The use of the Bethesda terminology in thyroid fine-needle aspiration results in a lower rate of surgery for nonmalignant nodules: a re- port from a reference center in Turkey. Int J Surg Pathol. 2011;19(6):761-771.

25. Lee KH, Shin JH, Oh YL, Hahn SY. Atypia of undetermined significance in thyroid fine-needle aspiration cytology: prediction of malignancy by US and comparison of methods for further management. Ann Surg Oncol. 2014;21(7):2326-2331.

26. Valderrabano P, Khazai L, Thompson ZJ, Sharpe SC, Tarasova VD, Otto KJ, Hallanger-Johnson JE, et al. Cancer risk associated with nuclear atypia in cytologically indeterminate thyroid nodules: a systematic review and meta-analysis. Thyroid. 2018;28(2):210-219.

27. Allen L, Al Afif A, Rigby MH, Bullock MJ, Trites J, Taylor SM, Hart RD. The role of repeat fine needle aspiration in managing indeterminate thyroid nodules. J Otolaryngol Head Neck Surg. 2019;48(1):16.

28. VanderLaan PA, Marqusee E, Krane JF. Clinical outcome for atypia of undetermined significance in thyroid fineneedle aspirations: should repeated fna be the preferred initial approach? Am J Clin Pathol. 2011;135(5):770-775. 